University of Nebraska - Lincoln

DigitalCommons@University of Nebraska - Lincoln

MODELING DYNAMIC FRACTURE AND DAMAGE IN A FIBERREINFORCED COMPOSITE LAMINA WITH PERIDYNAMICS

Wenke Hu

University of Nebraska-Lincoln

Youn Doh $\mathrm{Ha}$

Kunsan National University, Korea

Florin Bobaru

University of Nebraska-Lincoln, fbobaru2@unl.edu

Follow this and additional works at: https://digitalcommons.unl.edu/mechengfacpub

Hu, Wenke; Ha, Youn Doh; and Bobaru, Florin, "MODELING DYNAMIC FRACTURE AND DAMAGE IN A FIBER-REINFORCED COMPOSITE LAMINA WITH PERIDYNAMICS" (2011). Mechanical \& Materials Engineering Faculty Publications. 97.

https://digitalcommons.unl.edu/mechengfacpub/97

This Article is brought to you for free and open access by the Mechanical \& Materials Engineering, Department of at DigitalCommons@University of Nebraska - Lincoln. It has been accepted for inclusion in Mechanical \& Materials Engineering Faculty Publications by an authorized administrator of DigitalCommons@University of Nebraska Lincoln. 


\title{
MODELING DYNAMIC FRACTURE AND DAMAGE IN A FIBER-REINFORCED COMPOSITE LAMINA WITH PERIDYNAMICS
}

\author{
Wenke $\mathrm{Hu}_{1}{ }^{1}$ Youn Doh $\mathrm{Ha}^{1,2}$ \& Florin Bobaru, ${ }^{1, *}$ \\ ${ }^{1}$ Department of Mechanical and Materials Engineering, University of Nebraska-Lincoln, Lincoln, \\ Nebraska 68588-0526, USA \\ ${ }^{2}$ Assistant Professor, Department of Naval Architecture, Kunsan National University, Korea \\ *Address all correspondence to Florin Bobaru, E-mail: fbobaru2@unl.edu
}

We propose a peridynamic formulation for a unidirectional fiber-reinforced composite lamina based on homogenization and mapping between elastic and fracture parameters of the micro-scale peridynamic bonds and the macro-scale parameters of the composite. The model is then used to analyze the splitting mode (mode II) fracture in dynamic loading of a $0^{\circ}$ lamina. Appropriate scaling factors are used in the model in order to have the elastic strain energy, for a fixed nonlocal interaction distance (the peridynamic horizon), match the classical one. No special criteria for splitting failure are required to capture this fracture mode in the lamina. Convergence studies under uniform grid refinement for a fixed horizon size (m-convergence) and under decreasing the peridynamic horizon (ס-convergence) are performed. The computational results show that the splitting fracture mode obtained with peridynamics compares well with experimental observations. Moreover, in the limit of the horizon going to zero, the maximum crack propagation speed computed with peridynamics approaches the value obtained from an analytical classical formulation for the steady-state dynamic interface debonding found in the literature.

KEY WORDS: fiber-reinforced composites, dynamic fracture, damage, peridynamics, multi-scale modeling

\section{INTRODUCTION}

\subsection{Literature Review}

Two main fracture modes observed in breaking fiber-reinforced composite laminates are interlamina (between the plies) and intralamina (inside a ply) fracture. For intralamina fracture we distinguish between three types of fracture modes: splitting fracture (debonding between the fibers and the matrix), matrix cracking, and fiber breakage. For a unidirectional (UD) fiber-reinforced lamina under tensile loading, cracks will initiate at regions of high stress concentrations, such as the tip of a notch, and propagate along the fiber direction through splitting and matrix cracking. This fracture mechanism was experimentally studied, primarily under quasi-static loading, by Wu (1968), Kortschot and Beaumont (1990a,b), Bogert et al. (2006), and Hallett et al. (2009). Wu (1968) tested UD composites with center cracks in the direction of the fibers under three different loading types: tension, pure shear, and combined tension and shear. In all these tests, the crack always propagated in a direction co-linear with the original crack. Kortschot and Beaumont $(1990 \mathrm{a}, \mathrm{b})$ used double-edge-notched specimens $(0 / 90)_{s}$ tested under the tensile loading and observed, through radiographs of these specimens, splitting fracture mode in $0^{\circ}$ plies. Similar experimental results were obtained by Bogert et al. (2006) with uniaxial tension tests of UD center-notch specimens with $0^{\circ}$ plies. Recently, Hallett et al. (2009) investigated the damage evolution in composite laminates with a circular hole at different static load levels. The splits propagated in a symmetric manner in the $0^{\circ}$ plies.

Considerably fewer experiments have been reported in the literature on dynamic tensile loading of fiber-reinforced composites. There are some difficulties when performing experiments in composite materials with dynamic tensile 
loadings. Generating a tensile pulse and specimen gripping (Eskandari and Nemes, 2000) are two of the difficulties in dynamic tensile loading of composites. Because of these difficulties, the impact test has been the most common experimental method used to study the dynamic crack propagation behavior in composite materials. Recently, Lee et al. (2009) studied the damage growth in composites under impact loading. The crack propagation behavior is similar to the crack growth under static tensile loading. Kazemahvazi et al. (2009) induced dynamic tensile loadings in composite laminates via projectile impact. The fracture behavior under these conditions was studied at different strain rates. Multiple splits were observed in $0^{\circ}$ ply. The departure from the damage behavior under quasi-static loading was dramatic (Kazemahvazi et al., 2009). Ravi et al. (2001) reported experimental results in which the dynamic tensile loading in fiber-reinforced laminated composites was created through explosions. The results showed splitting and crack branching in the matrix component when the initial notch was orientated parallel to the fiber direction. This, again, is a behavior significantly different from the quasi-static loading conditions.

Significant efforts have been made to develop numerical models that can predict the strength of fiber-reinforced composites and model crack propagation in these materials. Most of these developments have been made for quasistatic type loading. Numerical studies based on the finite-element method (FEM) have been performed to compute the response of composite panels containing a notch or holes. Explicit damage models are need in these formulations and are usually based on cohesive-zone models (Jiang et al., 2007), or Schapery's Theory (Pineda, 2009). They simulated the split mode by FEM simulation in center-notched laminates for $0^{\circ}$ layers under uniaxial tension. However, during these numerical simulations, prior knowledge of the damage path and special techniques are required to predict the damage mode. The damage pattern may not be captured correctly if the split elements are misplaced. Also, a small amount of fiber failure occurred during the simulation done by Pineda et al. (2009), but not observed in experimental results (Bogert et al., 2006). These traditional methods, which are based on classical continuum mechanics, cannot directly be applied to problems with discontinuous fields. Thus, additional criteria are required to evaluate the spatial derivatives on the crack tip or crack surface.

The peridynamic model (Silling, 2000), unlike the formulations mentioned above, which are based on classical continuum mechanics models, does not need ad hoc criteria to guide the dynamic crack propagation. Recently, two peridynamic models were proposed for modeling composites by Xu et al. (2007, 2008), and Kilic et al. (2009).

The model used in Xu et al. (2008) is based on a homogenization approach in which the stiffness of the peridynamic bonds parallel to the fiber direction is fitted (or calibrated) to the elastic modulus of the lamina in the same direction. All other bonds have their stiffness fitted to lamina properties along the direction perpendicular to the fiber direction. For a brief introduction to peridynamics and a definition of peridynamic bonds see Section 2. Using this model, damage and failure modes of laminated composite plates under biaxial loading were simulated by Xu et al. (2007). The crack patterns with different notch orientations agreed well with experimental results. This model was also utilized to predict damage patterns in laminated composites subjected to low-velocity impact (Xu et al., 2008). The matrix damage and delamination patterns with respect to the impact energy from the peridynamics simulation agreed well with the experimental results. We note that in this peridynamic model for composites the evaluation of the bond stiffness is done via a computational procedure for a specific horizon and a specific discretization size, and analytical formulas for the parameters used in the model are not given. The model can be viewed as a "discretize, then calibrate" scheme. Even though this is not specified in their papers, it appears that the reason for calibrating the discretized model to the bulk elastic properties is to compensate for numerical discretization errors. Another approach is to "calibrate, then discretize," as described by Silling and Askari (2005) for the isotropic case, and this approach will be developed in the present paper for anisotropic materials. With the model presented here we discuss the issues of convergence of the crack path in terms of the grid size and horizon size, the " $m$-convergence" and the " $\delta$-convergence" (see Bobaru et al., 2009). We provide analytical formulas to obtain the peridynamic parameters for the continuous model and its discretization.

Kilic et al. (2009) explicitly modeled individual fibers. Bond stiffness for bonds inside the fibers was fitted to fiber properties, while bond stiffness for bonds inside the matrix material was fitted to matrix properties using the Silling and Askari (2005) procedure for an isotropic material. This model has been applied to simulate the damage of center-notched UD composite and laminates under quasi-static loading (Kilic et al., 2009). The results matched well with some quasi-static experiments. However, this model has significant limitations since it is not practical for solving realistic problems in fiber-reinforced laminated composites because of the huge number of fibers contained 
in such materials. Our results will show that, contrary to the statements made by Kilic et al. (2009), it is possible to successfully use homogenization to model fracture and damage in fiber-reinforced composites using peridynamics.

In the present paper, we propose a homogenization-based peridynamic model for simulating fracture and damage in fiber-reinforced composites and study the convergence behavior for loading cases that lead to splitting facture in a $0^{\circ}$ lamina. We provide analytic formulas to obtain the bond stiffness for bonds aligned with the fiber direction [called "fiber bonds" (fb)] and all other bonds [called "matrix bonds" (mb)]. These formulas are obtained by matching the peridynamic strain energy density in a composite lamina under a homogeneous deformation with the classical linear elastic strain energy density under the same deformation. The homogenization scheme allows us to connect the properties at the micro-scale in the peridynamic model for any horizon to the macro-scale properties of the composite. We also obtain certain scaling factors for the bond stiffness of fiber bonds and matrix bonds in order to have the elastic strain energy, for a given horizon, match the classical elastic strain energy. Two types of convergence were performed for the splitting crack propagation by refining the grid and decreasing the horizon size (Bobaru et al., 2009). The crack paths from our simulations are similar to those observed in experiments. The computed crack propagation velocity, in the limit of the horizon going to zero, approaches values analytically obtained by Yang et al. (1991) with a local model for a steady-state propagating crack in interface debonding.

The paper is organized as follows: in Sections 2 and 3, we briefly review the basic formulation for peridynamics and the classical equations for a UD fiber-reinforced composite lamina. In Section 4 we present the new multiscale model with the analytical connections between the microscopic parameters in the peridynamic formulation and the macroscopic material properties, as well as obtain the scaling factors required for maintaining the same elastic strain energy density with a classical material. In Section 5 we present the numerical results for splitting modes in a UD composite lamina and perform convergence studies in terms of the crack path and crack propagation velocity. Conclusions are given in the final Section 6.

\section{REVIEW OF PERIDYNAMICS}

The peridynamic theory (Silling, 2000) is a non-local formulation that extends the classical continuum mechanics formulation. In peridynamics, every material point is connected to the other points inside a certain "horizon" region through peridynamic bonds. In this way, instead of the divergence of stress terms in the classical equations, one uses an integral over the horizon of the current point of forces, per unit volume squared, acting in the peridynamic bonds. Since the spatial differentiation is eliminated from the mathematical framework of peridynamics, this formulation is well suited to modeling problems in which discontinuities emerge, interact, and evolve in time. Indeed, the integration of forces can be directly applied over discontinuous displacement fields, which appear in the case of crack propagation. The peridynamic equations of motion are given as

$$
\rho \ddot{\mathbf{u}}(\mathbf{x}, \mathrm{t})=\int_{H} \mathbf{f}[\mathbf{u}(\mathbf{x}, \mathrm{t})-\mathbf{u}(\mathbf{x}, \mathrm{t}), \hat{\mathbf{x}}-\mathbf{x}] \mathrm{dV}_{\hat{\mathbf{x}}}+\mathbf{b}(\mathbf{x}, \mathrm{t})
$$

where $\mathbf{f}$ is the pairwise force function in the peridynamic bond that connects point $\hat{\mathbf{x}}$ to $\mathbf{x} ; \mathbf{u}$ is the displacement vector field; $\rho$ is the density; and $\mathbf{b}(\mathbf{x}, t)$ is the body force. The integral is defined over a region $H$ called the horizon. The region is taken here to be a circle of radius $\delta$, but its shape is arbitrary. The horizon is the compact supported domain of the pairwise force function around a point $\mathbf{x}$. We will abuse the terminology and also call the horizon the radius of the horizon, $\delta$.

In principle, the exact size and shape of the horizon could be found from wave dispersion curves for a specific material under specific dynamic conditions (see Silling, 2000). In practice, for problems where a material length scale does not readily manifests itself, we take the horizon to smaller and smaller values and monitor the convergence of the results (see, e.g., Bobaru et al., 2009; Ha and Bobaru, 2010). A convenient horizon size is one sufficiently large such that the computations are efficient (we can use coarser grids with a larger horizon), but sufficiently small such that the results do not change much if one chooses to use a smaller horizon. Please note that in dynamic fracture problems, while one cannot guarantee convergence of results in the limit of the horizon going to zero, recent simulations show for crack branching problems that convergence in terms of the crack path and crack propagation speed happens as the horizon goes to zero (see, e.g., Ha and Bobaru 2010; Ha and Bobaru, 2011a, 2011b). Let $\xi=\hat{\mathbf{x}}-\mathbf{x}$ be the relative 
position in the reference configuration and $\eta=\hat{\mathbf{u}}-\mathbf{u}$ be the relative displacement. From the definition of the horizon, we have

$$
\|\xi\|>\delta \Rightarrow \mathbf{f}(\eta, \xi)=0
$$

so that no force acts between two material points if the relative position (in the reference configuration) is larger than the given horizon size.

A micro-elastic material (Silling, 2000) is defined as one in which the pairwise force derives from a potential $\omega$ :

$$
\mathbf{f}(\eta, \xi)=\frac{\partial \omega(\eta, \xi)}{\partial \eta}
$$

A "linear" micro-elastic potential assumes a linear relationship between the bond force and the relative elongation of the bond:

$$
\omega(\eta, \xi)=\frac{c(\xi) s^{2} \xi}{2}
$$

where $\xi=\|\xi\|$ and

$$
s=\frac{\|\xi+\eta\|-\xi}{\xi}
$$

and where $s$ is the bond relative elongation.

The corresponding pairwise force becomes

$$
\mathbf{f}(\eta, \xi)=\frac{\partial \omega(\eta, \xi)}{\partial \eta}=c(\xi) s\|\xi\| \frac{\partial s}{\partial \eta}=c(\xi) s \frac{\partial\|\xi+\eta\|}{\partial \eta}
$$

with

$$
\frac{\partial\|\xi+\eta\|}{\partial \eta}=\frac{\xi+\eta}{\|\xi+\eta\|}=\mathbf{e}
$$

where $\mathbf{e}$ is the unit vector along the direction of the bond between $\hat{\mathbf{x}}$ and $\mathbf{x}$ in the deformed configuration $(\xi+\eta)$.

The function $\mathrm{c}(\xi)$ is called the micromodulus function and it represents the bond elastic stiffness, which can be linear or nonlinear. This function is required to satisfy certain conditions of regularity; however, the set of allowable functions is quite large (Silling et al., 2003). For the isotropic material, the various micromodulus functions are given in one-dimensional (1D) (Bobaru et al., 2009), two-dimensional (2D) (Ha and Bobaru, 2010) and three-dimensional (3D) (Silling and Askari, 2005) versions. In all of these cases, the micromodulus function is obtained by matching the strain energy density in peridynamics to the strain energy density in the classical theory under the same homogeneous deformation.

\section{CLASSICAL MECHANICS OF A UNIDIRECTIONAL COMPOSITE LAMINA IN TWO DIMENSIONS}

A UD composite lamina is a planar arrangement of unidirectional fibers strongly bonded in a matrix. At the macromechanical level, the fiber-reinforced UD composite is an orthotropic, homogeneous, linearly elastic material (Cristescu et al., 2004). Thus, for the lamina the constitutive equation can be written in the following matrix form:

$$
\left[\begin{array}{c}
\sigma_{1} \\
\sigma_{2} \\
\sigma_{3} \\
\sigma_{4} \\
\sigma_{5} \\
\sigma_{6}
\end{array}\right]=\left[\begin{array}{cccccc}
C_{11} & C_{12} & C_{13} & 0 & 0 & 0 \\
C_{12} & C_{22} & C_{23} & 0 & 0 & 0 \\
C_{13} & C_{23} & C_{33} & 0 & 0 & 0 \\
0 & 0 & 0 & C_{44} & 0 & 0 \\
0 & 0 & 0 & 0 & C_{55} & 0 \\
0 & 0 & 0 & 0 & 0 & C_{66}
\end{array}\right]\left[\begin{array}{c}
\varepsilon_{1} \\
\varepsilon_{2} \\
\varepsilon_{3} \\
\varepsilon_{4} \\
\varepsilon_{5} \\
\varepsilon_{6}
\end{array}\right]
$$

where the Voigt notation is used for a single subscript notation for stress and strain. 
In this research, we will use a 2D plane stress model. The 2D plane stress constitutive equation for UD composites can be written as

$$
\left[\begin{array}{l}
\sigma_{1} \\
\sigma_{2} \\
\sigma_{3}
\end{array}\right]=\left[\begin{array}{ccc}
C_{11} & C_{12} & 0 \\
C_{12} & C_{22} & 0 \\
0 & 0 & C_{66}
\end{array}\right]\left[\begin{array}{l}
\varepsilon_{1} \\
\varepsilon_{2} \\
\varepsilon_{3}
\end{array}\right]
$$

where

$$
C_{11}=\frac{E_{11}}{1-v_{12} v_{21}}, \quad C_{22}=\frac{E_{22}}{1-v_{12} v_{21}}, \quad C_{12}=\frac{v_{21} E_{11}}{1-v_{12} v_{21}}, \quad C_{66}=G_{12}
$$

Connections between the properties of the fibers and the matrix and the effective properties of the lamina can be expressed, for example, by using the Halpin-Tsai relationships (Halpin and Kardos, 1976):

$$
\begin{array}{cc}
\mathrm{E}_{11}=\mathrm{V}_{\text {fiber }} \mathrm{E}_{\text {fiber }}+\mathrm{V}_{\text {matrix }} \mathrm{E}_{\text {matrix }}, & \frac{1}{\mathrm{E}_{22}}=\frac{\mathrm{V}_{\text {fiber }}}{\mathrm{E}_{\text {fiber }}}+\frac{\mathrm{V}_{\text {matrix }}}{\mathrm{E}_{\text {matrix }}} \\
v_{12}=\mathrm{V}_{\text {fiber }} v_{\text {fiber }}+\mathrm{V}_{\text {matrix }} v_{\text {matrix }}, & \mathrm{V}_{\text {fiber }}+\mathrm{V}_{\text {matrix }}=1
\end{array}
$$

where $\mathrm{E}_{11}$ and $\mathrm{E}_{22}$ are the longitudinal and transverse elastic Young's modulus in the principal material axes, respectively; $v_{12}$ is the longitudinal Poisson's ratio and $v_{21}$ is the transverse Poisson's ratio; $G_{12}$ is the elastic shear modulus; and $\mathrm{E}_{\mathrm{fiber}}, \mathrm{E}_{\text {matrix }}, v_{\text {fiber }}, v_{\text {matrix }}, \mathrm{V}_{\text {fiber }}$, and $\mathrm{V}_{\text {matrix }}$ are the fiber and matrix moduli, fiber and matrix Poisson's ratio, fiber volume fraction, and matrix volume fraction, respectively.

\section{THE NEW PERIDYNAMIC MODEL FOR A UNIDIRECTIONAL COMPOSITE LAMINA}

As mentioned in the Introduction, the peridynamic model given by Kilic et al. (2009) for fiber-reinforced composites has significant limitations: the explicit modeling of individual fiber regions is not an option once the number of fibers in the composite is large (which is always the case). The model proposed by Silling and co-workers (Xu et al., 2007, 2008) and implemented in the EMU code from Sandia National Laboratories, requires a numerical evaluation of the parameters in the model for every particular grid used based on matching the strain energy for a homogeneous deformation with a classical elastic composite material (the discretize, then calibrate scheme). In this model (Xu et al., 2008), for every horizon and grid spacing used one performs a preliminary computation to determine the peridynamic parameters to be used in that case so that the total strain energy under a homogeneous deformation of the specific computational model matches the one corresponding to an "equivalent" classical elastic composite. Analytical expressions for this model are not given.

In this section, we present a new formulation for modeling fiber-reinforced composites, which differs from the one given by Xu et al. (2008) in the sense that, here, we obtain analytical formulas for the bond elasticity via a homogenization approach and the model is discretized only afterward. We obtain formulas for the micromodulus function and the micro-damage parameters (the critical relative elongations) that can be directly used in computations without a preliminary numerical evaluation of the solution of the problem. We evaluate scaling factors for the peridynamic material micro-scale parameters corresponding to the continuous model as well as for a specific computational grid so that the peridynamic elastic strain energy density matches the corresponding classical energy. The new model is based on a homogenization procedure similar to the one performed for isotropic materials but applied here for the elastic deformation of the UD composite lamina. We obtain the peridynamic constitutive relations for the fiber bonds and matrix bonds by relating them to the different properties of the UD composite lamina along the longitudinal and transverse directions.

Remark: Please note that in our model the peridynamic fiber bonds are not matched to the actual fiber properties, but are calibrated to the effective elastic properties of the lamina along the fiber direction. The same is true for the matrix bonds. In other words, the micromoduli of fiber bonds and matrix bonds are obtained from, respectively, matching the peridynamic strain energy to the classical strain energy along the longitudinal and transverse directions of the lamina. This description of peridynamic bonds is similar to that used in the model presented by Xu et al. (2008) but it is different from the explicit modeling of fibers used by Kilic et al. (2009). 


\subsection{The Peridynamic Model for a Composite Lamina}

We assume that strain energy in the longitudinal direction is a result of contributions from peridynamic fiber bonds while the strain energy along the transverse direction comes from the matrix bonds. To arrive at a homogenized model for the lamina we follow the procedure schematically shown in Fig. 1. The peridynamic bonds at a point in the anisotropic peridynamic material will have different properties along the longitudinal direction than along all other directions. To compute the strain energy density along the longitudinal deformation we need to make use of the Dirac-delta function. For a given homogeneous deformation, we match the strain energy of the material along the longitudinal direction with the strain energy of homogenized anisotropic peridynamic material along the same direction. Along the transverse direction we do the same.

Given a homogeneous bi-axial deformation of size $s$ aligned with the longitudinal and the transverse directions, the peridynamic elastic strain energy density of the UD composite lamina can be written as

$$
\begin{gathered}
\mathrm{W}_{1}=\frac{1}{2} \int_{H} \omega_{\mathrm{fb}}(\eta, \xi)[\mathrm{D}(\xi, 0)+\mathrm{D}(\xi, \pi)] \mathrm{dA} \xi=\frac{1}{2} \int_{-\delta}^{\delta} \omega_{\mathrm{fb}}(\eta, \xi) d \xi \\
\mathrm{W}_{2}=\frac{1}{2} \int_{H} \omega_{\mathrm{mb}}(\eta, \xi) \mathrm{dA} \xi \\
\omega_{\mathrm{fb}}(\eta, \xi)=\frac{\bar{c}_{\mathrm{fb}}(\xi) \mathrm{s}^{2} \xi}{2}, \omega_{\mathrm{mb}}(\eta, \xi)=\frac{\bar{c}_{\mathrm{mb}}(\xi) \mathrm{s}^{2} \xi}{2} \omega_{\mathrm{mb}}(\eta, \xi)=\frac{\bar{c}_{\mathrm{mb}}(\xi) \mathrm{s}^{2} \xi}{2}
\end{gathered}
$$

where $s$ is the constant strain value of the homogeneous deformation [also see Eq. (5)]; $\omega_{\mathrm{fb}}$ and $\omega_{\mathrm{mb}}$ are the microelastic potentials for the anisotropic peridynamic material along the longitudinal and transverse directions; $\bar{c}_{\mathrm{fb}}$ and $\bar{c}_{\mathrm{mb}}$ are the elastic stiffness (micromodulus) functions for peridynamic bonds along the longitudinal direction and the transverse direction, respectively; and $\mathrm{D}(\xi, 0)$ and $\mathrm{D}(\xi, \pi)$ are the 2D Dirac-delta functions (distribution) for the polar coordinates $\theta=0$ and $\theta=\pi$ (where $\theta=\arctan \left(\xi_{2} / \xi_{1}\right), \xi=\left(\xi_{1}, \xi_{2}\right)=(\xi, \theta)$ ).

We impose the peridynamic elastic strain energy densities above to equal the longitudinal and transverse components, respectively, of the strain energy density under 2D plane stress conditions of a classical elasticity composite material under the same homogenous deformation. The classical strain energy density for the homogenized composite lamina is given by

$$
\mathrm{W}_{1}^{\text {classical }}=\frac{1}{2} \frac{\left(\mathrm{E}_{11}+v_{12} \mathrm{E}_{22}\right)}{\left(1-v_{12} v_{21}\right)} \mathrm{s}^{2}, \quad \mathrm{~W}_{2}^{\text {classical }}=\frac{1}{2} \frac{\left(\mathrm{E}_{22}+v_{12} \mathrm{E}_{22}\right)}{\left(1-v_{12} v_{21}\right)} \mathrm{s}^{2}
$$

We use here the "conical" micromodulus function (see Ha and Bobaru, 2010) because this function gives a slightly smaller error against the classical solution in the limit of the horizon going to zero, compared with the constant

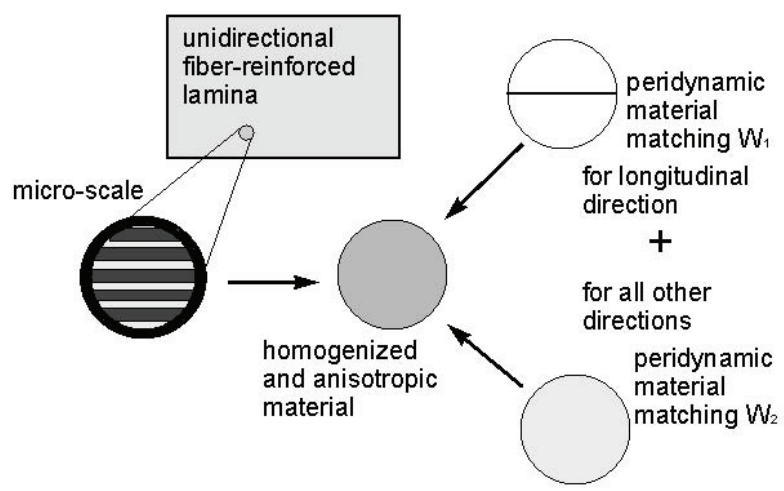

FIG. 1: Schematic for the procedure to obtain the homogenized anisotropic peridynamic material 
micromodulus function (Bobaru et al., 2009; Ha and Bobaru, 2010; Ha et al. 2010). By matching the corresponding elastic strain energies, for the peridynamic fiber bonds we get

$$
\bar{c}_{\mathrm{fb}}=\frac{6\left(\mathrm{E}_{11}+v_{12} \mathrm{E}_{22}\right)}{\left(1-v_{12} v_{21}\right) \delta^{2}}\left(1-\frac{\xi}{\delta}\right)
$$

while for bonds along the transverse direction (matrix bonds) we obtain

$$
\bar{c}_{\mathrm{mb}}=\frac{12\left(\mathrm{E}_{22}+v_{12} \mathrm{E}_{22}\right)}{\left(1-v_{12} v_{21}\right) \pi \delta^{3}}\left(1-\frac{\xi}{\delta}\right)
$$

The micromodulus function for the homogenized anisotropic peridynamic material, therefore, is (see Fig. 2):

$$
\mathrm{C}(\xi, \theta)=\left\{\begin{array}{lr}
\bar{c}_{\mathrm{fb}}(\xi) & \text { if } \theta=0 \text { or } \pi \\
\bar{c}_{\mathrm{mb}}(\xi) & \text { otherwise }
\end{array}\right.
$$

Therefore, the pairwise force function $\mathbf{f}$ in Eq. (1) can write as follows

$$
\mathbf{f}(\eta, \xi)=\left\{\begin{array}{lll}
\mathbf{f}_{\mathrm{fb}}(\eta, \xi)[D(\xi, 0)+D(\xi, \pi)] & \text { if } \theta=0 \text { or } \pi \\
\mathbf{f}_{\mathrm{mb}}(\eta, \xi) & \text { otherwise } &
\end{array}\right.
$$

where $\mathbf{f}_{\mathrm{fb}}(\eta, \xi)=\overline{\mathrm{c}}_{\mathrm{fb}}(\xi) \mathrm{s}(\partial\|\xi+\eta\|) /(\partial \eta)$ and $\mathbf{f}_{\mathrm{fb}}(\eta, \xi)=\bar{c}_{\mathrm{mb}}(\xi) \mathrm{s}(\partial\|\xi+\eta\|) /(\partial \eta)$, with s given in Eq. (5).

Notice that if we were to consider an isotropic peridynamic material that would match $\mathrm{W}_{1}$, the micromodulus for such a material would have been

$$
c_{11}^{\text {iso }}=\frac{12\left(E_{11}+v_{12} E_{22}\right)}{\left(1-v_{12} v_{21}\right) \pi \delta^{3}}\left(1-\frac{\xi}{\delta}\right)
$$

Similarly, for the transverse direction, to match $\mathrm{W}_{2}$, we would get

$$
c_{22}^{\text {iso }}=\frac{12\left(E_{22}+v_{12} E_{22}\right)}{\left(1-v_{12} v_{21}\right) \pi \delta^{3}}\left(1-\frac{\xi}{\delta}\right)
$$

Observe that the ratio between $\bar{c}_{\mathrm{fb}}$ and $\mathrm{c}_{11}^{\text {iso }}$ is $\pi \delta / 2$. Notice also that $\mathrm{c}_{22}^{\text {iso }}$ is the same as $\bar{c}_{\mathrm{mb}}$.

In the bond-based peridynamics the Poisson ratios and the shear modulus are set by the selection of fiber and matrix bonds stiffness. For the Poisson's ratio $v_{12}$ in Eqs. (14), (15), (17), and (18), we use a value of 1/3. Because of the dependency mentioned, the actual value for this parameter may be different from this. In an isotropic material the 1/3
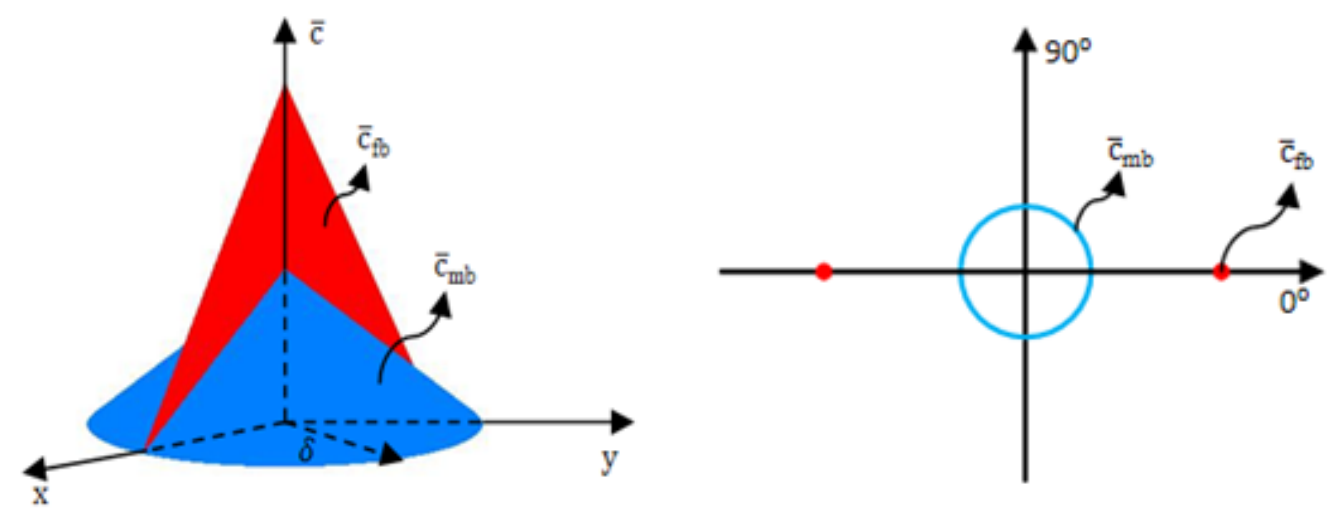

FIG. 2: Conical micromodulus function for the UD composite lamina (left) at a point in the bulk. The variation of the micromodulus at a fixed distance from the node, with respect to the angle between the bond and the fiber direction (right). 
value is the resulting Poisson ratio for a bond-based peridynamic model in two dimensions. Because of the symmetry of the behavior for an orthotropic material, for the other Poisson ratio $v_{21}$ we employ the following reciprocal relation:

$$
v_{21}=\frac{E_{22} v_{12}}{E_{11}}
$$

Note that the shear modulus does not enter specifically in the formulation. Its value will be dependent on the elastic moduli and the Poisson ratios. We mention that in the state-based peridynamics (Silling et al., 2007) the Poisson ratios and the shear modulus values can be independently modeled.

\subsection{The Peridynamic Micro-Damage Model for Fracture and Damage in a UD Composite Lamina}

The damage model in peridynamics (Silling, 2000) consists in breaking the peridynamic bonds connecting any two nodes when the relative change in distance between the nodes exceeds a certain prescribed value. This critical relative elongation parameter $\mathrm{s}_{0}$ is obtained by equating the work, per unit fracture area, required to break all the bonds across the fracture surface to the fracture energy required for complete separation of the two halves of the body (Silling and Askari, 2005).

We first briefly review the formulation of damage in peridynamics. As mentioned by Silling and Askari (2005), the pairwise force for a micro-elastic material with damage is

$$
\mathrm{f}(\eta, \xi)=\frac{\xi+\eta}{\|\xi+\eta\|} \mathrm{c}(\xi) s \mu(\mathrm{t}, \xi)
$$

where $\mu(t, \xi)$ is a history-dependent scalar-value function that has the value of either 0 or 1 :

$$
\mu(t, \xi)= \begin{cases}1 & \text { if } \mathrm{s}(t, \xi)<\mathrm{s}_{0} \\ 0 & \text { if } \mathrm{s}(t, \xi)>\mathrm{s}_{0}\end{cases}
$$

The model is now history dependent. The bonds cannot sustain any force after breaking and this procedure is irreversible. Reversible-type damage can also be introduced (Silling and Bobaru, 2005; Bobaru, 2007) and allow bonds to re-form.

In an isotropic and homogeneous material, the critical relative elongation $\mathrm{s}_{0}$ at the micro-scale can be obtained from the macro-scale measureable fracture energy $\left(\mathrm{G}_{0}\right)$. In two dimensions (Ha and Bobaru, 2010), the fracture energy is

$$
\mathrm{G}_{0}=2 \int_{0}^{\delta} \int_{\mathrm{z}}^{\delta} \int_{0}^{\cos ^{-1}(\mathrm{Z} / \xi)}\left[\mathrm{c}_{\mathrm{iso}}(\xi) \mathrm{s}_{0}^{2} \xi / 2\right] \xi d \theta d \xi d z
$$

where $c_{\text {iso }}$ is the micromodulus of the isotropic material and the integration domain is shown in Fig. 3 as given by Ha and Bobaru (2010). The 3D form is given by Silling and Askari (2005). The critical relative elongation $\mathrm{s}_{0}$ can be obtained from the above equation.

Next, we introduce our damage model for UD composites. The simplest way to introduce failure into the model is by allowing fiber bonds and all other bonds (matrix bonds) to break when they are stretched beyond a critical relative elongation, $\mathrm{s}_{0}^{\mathrm{fb}}$ and $\mathrm{s}_{0}^{\mathrm{mb}}$. The critical relative elongation $\mathrm{s}_{0}^{\mathrm{fb}}$ is obtained by matching the work needed to break all bonds in a material made entirely out of fiber bonds (not fibers), across a line (for the $2 \mathrm{D}$ case) to the fracture energy $\mathrm{G}_{0}^{11}$. The value for $\mathrm{s}_{0}^{\mathrm{mb}}$ can be obtained in a similar way by matching $\mathrm{G}_{0}^{22}$ with the work needed to break all bonds in a material made entirely out of matrix bonds (not matrix material), across a line (in two dimensions). The values we obtain are

$$
\mathrm{s}_{0}^{\mathrm{fb}}=\sqrt{\frac{20 \mathrm{G}_{0}^{11}}{\mathrm{c}_{11}^{\text {iso }} \delta^{4}}}, \quad \mathrm{~s}_{0}^{\mathrm{mb}}=\sqrt{\frac{20 \mathrm{G}_{0}^{22}}{\mathrm{c}_{22}^{\text {iso }} \delta^{4}}}
$$

where $\mathrm{G}_{0}^{11}$ and $\mathrm{G}_{0}^{22}$ are the fracture energies for a UD composite with $0^{\circ}$ fiber orientation and $90^{\circ}$ fiber orientation lamina, respectively. Recall that the fiber bond properties are not matched to the fiber material, but to the effective properties along the fiber direction. The same goes for matrix bonds. 


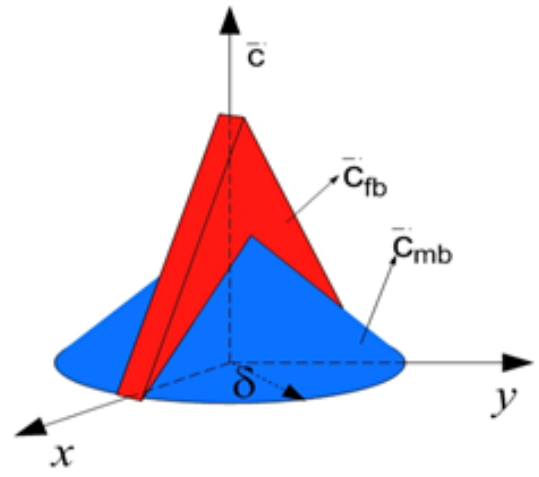

(a)

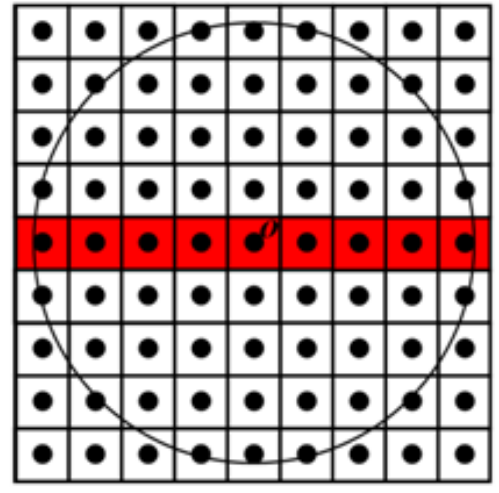

(b)

FIG. 3: Conical micromodulus function for the UD composite lamina at a point in the bulk (a). The discrete peridynamic model for unidirectional lamina at a particular node $\mathrm{O}(\mathbf{b})$. The circle is the horizon for this node. Fiber direction is horizontal in this example; thus, the peridynamic fiber bonds for the central node exist only with nodes having their areas colored in red (since only these bonds, centered at $\mathrm{O}$, have the same direction as the fiber direction).

The fracture energies $\mathrm{G}_{0}^{11}$ and $\mathrm{G}_{0}^{22}$ are experimentally measured from the intralaminar mode $\mathrm{I}$ fracture test (Jose et al., 2001). We emphasize that in this damage model, the critical relative elongation only depends on the material properties, $G_{0}^{11}, G_{0}^{22}, E_{11}, v_{12}$, and $E_{22}$, and also depend on the horizon size. Note that dependencies of the critical relative elongation on the current elastic deformation state, manufacturing defects, and time (Silling and Askari, 2005), or current damage state (Ha and Bobaru, 2010), can be easily introduced into the model if desired. The effect of the peridynamic material's response to these dependencies will be studied in the future.

\subsection{The Discrete Model and the Scaling of the Micromodulus Function}

In our model, the peridynamic fiber bonds emanating from a particular node are only those bonds that have the same or nearly the same direction as the longitudinal direction. All other bonds connected to this node are matrix bonds (see Fig. 2). Note that the discretization below is restricted to regular square grids with the principal directions aligned with the fiber direction, or to small perturbations of such grids. For random nodal arrangements or for grids not aligned with the fiber direction, the formulation is presented by $\mathrm{Hu}$ et al. (2011). The preference for uniform grids is due to the simplicity of generating them and conducting convergence tests, as well as the convenience for modeling the $0^{\circ}$ and $90^{\circ}$ fiber orientations, two of the most used in practice. Uniform grids aligned with the fiber direction are used in all of the computations in this paper.

In the discrete form of the peridynamic equation for the unidirectional fiber-reinforced lamina this singular function will be approximated by a sequence of functions that are zero outside the areas of nodes connected to the central node by fiber bonds. The micromodulus in Eq. (16) will, therefore, change because of the discretization. In what follows, for clarity, we directly derive these scaling factors for a given discretization based on matching the strain energy of the discrete peridynamic model to the classical longitudinal and transverse strain energies.

As a result of grid refinement for a fixed horizon size [the so-called $m$-convergence introduced by Bobaru et al. (2009), where $m$ is the ratio between the horizon and the grid spacing; see Ha and Bobaru (2010); see also Fig. 4], the fiber bond area in the peridynamic discretization at a node changes. This is schematically shown in Fig. 3(b), and the change is reflected in a change in the strain energy density at the node unless proper scaling is employed.

Let $\mathrm{W}_{1}^{\mathrm{d}}$ the elastic strain energy density computed over the fiber bond area in the discretized configuration in Fig. 3 based on the $\mathrm{c}_{11}^{\text {iso }}$ in Eq. (17). Obviously, this will not match $\mathrm{W}_{1}$ since we do not integrate over the entire horizon area. Similarly, let $\mathrm{W}_{2}$ be the elastic strain energy density computed over the matrix bond area in the discretized 


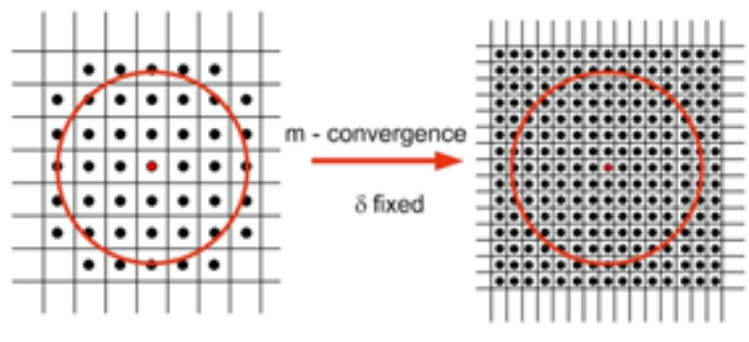

(a)

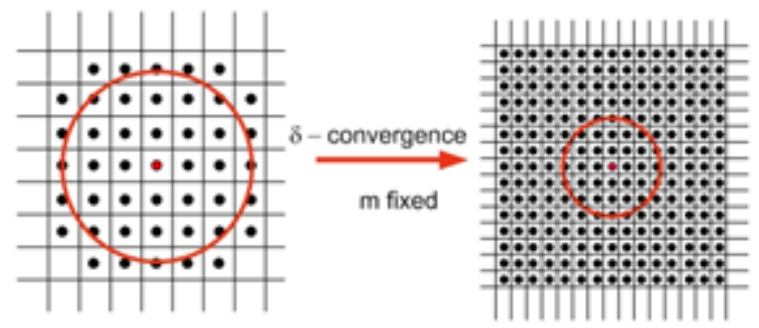

(b)

FIG. 4: Graphical description for the $m$-convergence and $\delta$-convergence. The ratio of the horizon $\delta$ to the grid spacing $\Delta x$ is $m$.

configuration in Fig. 3 based on the $c_{22}^{\text {iso }}$ in Eq. (18). We find the scaling factors $\lambda_{\mathrm{fb}}$ and $\lambda_{\mathrm{mb}}$ for the fiber bonds and matrix bonds in the discretized model, respectively, so that

$$
\lambda_{\mathrm{fb}} \mathrm{W}_{1}^{\mathrm{d}}=\mathrm{W}_{1}, \quad \lambda_{\mathrm{mb}} \mathrm{W}_{2}^{\mathrm{d}}=\mathrm{W}_{2}
$$

From Eqs. (10), (11), (23), and (24), the scaling factors for fiber bonds and matrix bonds are found as

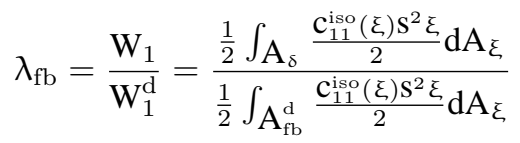

$$
\begin{aligned}
& \lambda_{\mathrm{mb}}=\frac{\mathrm{W}_{2}}{\mathrm{~W}_{2}^{\mathrm{d}}}=\frac{\frac{1}{2} \int_{\mathrm{A}_{\delta}} \frac{\mathrm{c}_{22}^{\text {iso }(\xi) \mathrm{S}^{2} \xi}}{2} \mathrm{dA}_{\xi}}{\frac{1}{2} \int_{\mathrm{A}_{\mathrm{fb}}^{\mathrm{d}}} \frac{\mathrm{cis2}_{22}^{\text {iso }(\xi) \mathbf{S}^{2} \xi}}{2} \mathrm{dA}_{\xi}}
\end{aligned}
$$

where $A_{\delta}$ is the total area covered by the horizon of radius $\delta$ [see the circle area in Fig. 3(b)]; and $\mathrm{A}_{\mathrm{fb}}^{\mathrm{d}}$ and $\mathrm{A}_{\mathrm{mb}}^{\mathrm{d}}$ are the fiber bond area [see Fig. 3(b), the red area inside the circle] and matrix bond area (the white areas inside the circle) for the particular discretization, respectively.

The discrete version for the conical micromodulus function case is then written as

$$
\mathrm{C}^{d}(\xi, \theta)=\left\{\begin{array}{lr}
\lambda_{\mathrm{fb}} \mathrm{c}_{11}^{\text {iso }}(\xi) & \text { if } \theta=0 \text { or } \pi \\
\lambda_{\mathrm{mb}} \mathrm{c}_{22}^{\text {iso }}(\xi) & \text { otherwise }
\end{array}\right.
$$

We can also express this micromodulus function in terms of the continuum peridynamic anisotropic model in Eq. (16):

$$
\mathrm{C}^{d}(\xi, \theta)= \begin{cases}\bar{\lambda}_{\mathrm{fb}} \bar{c}_{\mathrm{fb}}(\xi) & \text { if } \theta=0 \text { or } \pi \\ \bar{\lambda}_{\mathrm{mb}} \bar{c}_{\mathrm{mb}}(\xi) & \text { otherwise }\end{cases}
$$

where $\bar{\lambda}_{\mathrm{fb}}=2 \lambda_{\mathrm{fb}} / \pi \delta$ and $\bar{\lambda}_{\mathrm{mb}}=\lambda_{\mathrm{mb}}$. Moreover, the scaling factor $\lambda_{\mathrm{fb}}$ is independent of the horizon size. Because of this, in practice we will use the scaling of the discrete micromodulus function in the form of Eq. (26).

Remark: in practice, for the conical micromodulus function, instead of the values for $\lambda_{\mathrm{fb}}$ and $\lambda_{\mathrm{mb}}$ obtained above, we use the following approximations:

$$
\tilde{\lambda}_{\mathrm{fb}}=\frac{\mathrm{A}_{\delta}}{\mathrm{A}_{\mathrm{fb}}^{d}} \cong \frac{\pi m}{2}, \quad \tilde{\lambda}_{\mathrm{mb}}=\frac{\mathrm{A}_{\delta}}{\mathrm{A}_{\mathrm{mb}}^{d}} \cong \frac{\pi m}{\pi m-2}
$$

where $m=\delta / \Delta x \delta$, with $\Delta x$ is the grid spacing in a uniform discretization. These ratios of areas are good approximations for the ratios of strain energies above because of the nature of the integrand functions in Eqs. (24) and (25): these functions are zero at the node and at the boundary of the horizon. These approximations are less than $1 \%$ in error for values of $m=5$ or larger (see Table 1), and they should not be used for the constant micromodulus function (see Ha et al., 2010). In general, the scaling parameters can be computed, for example, from Eqs. (24) and (25) using numerical integration. 
TABLE 1: Comparison between the approximate and analytical values for the scaling factor for the conical micromodulus function

\begin{tabular}{|c|c|c|c|c|c|c|}
\hline & $\lambda_{\mathrm{fb}}$ & $\tilde{\lambda}_{\mathrm{fb}}$ & Error $^{1}$ & $\lambda_{\mathrm{mb}}$ & $\tilde{\lambda}_{\mathrm{mb}}$ & Error $^{2}$ \\
\hline$m=4$ & 6.1974 & 6.2832 & $1.38 \%$ & 1.1924 & 1.1893 & $0.26 \%$ \\
\hline$m=5$ & 7.7764 & 7.8540 & $1 \%$ & 1.1475 & 1.1459 & $0.14 \%$ \\
\hline
\end{tabular}

${ }^{1}$ Relative error between approximate and analytical scaling factor for fiber bonds.

${ }^{2}$ Relative error between approximate and analytical scaling factor for matrix bonds.

\section{NUMERICAL STUDIES OF CONVERGENCE FOR DYNAMIC SPLITTING IN A COMPOSITE LAMINA}

In peridynamics, three types of convergence studies have been introduced by Bobaru et al. (2009): these are $\delta$ convergence (decrease the horizon size, while the number of nodes covered by a horizon is kept constant), $m$ convergence (increase $m$ while holding the horizon fixed), and $(\delta m)$-convergence (decrease horizon size as well as increase $m$ ). See Fig. 4 for the first two convergence types. In the present study, we perform $m$-convergence calculations and make observations related to the behavior of the solution in terms of the crack propagation speed under a changing $\delta$. As discussed by Ha and Bobaru (2010), for problems involving damage and fracture changing $\delta$ changes the size of the damage zone associated with a pre-crack. Therefore, $\delta$-convergence has to take this aspect into account.

Moreover, note that in the actual implementation we use, for computational efficiency, a mid-point integration rule for evaluating spatial integrals with a special algorithm for approximating the nodal areas inside the horizon of a node (see Bobaru and Ha, 2011). This approximation affects the results only near the boundary where the integrand in the peridynamic formulation for the conical micromodulus goes to zero. Therefore, the results should not be influenced much, as long as $m$ is larger than $\sim 4$.

For the remainder of this paper, all of the simulations are performed based on the conical micromodulus function.

\subsection{Problem Setup}

Consider a central-crack thin rectangular plate with dimensions $0.2 \mathrm{~m} \times 0.1 \mathrm{~m}$ (see Fig. 5). Along the left and right edges a uniform tensile load $\sigma=40 \mathrm{~Pa}$ is applied suddenly and maintained constant in time after that. The practical implementation of traction boundary conditions in peridynamics and convergence studies have been discussed by Ha and Bobaru (2009).

The composite material used here is the M55J/M18 carbon/epoxy (Jose et al., 2001). The elastic and fracture properties are given in Table 2. Notice that in the present bond-based peridynamic model, the only inputs in the model are the values of the Young's moduli and values for mode I fracture energies for $0^{\circ}$ and $90^{\circ}$ fiber orientations. The values of the Poisson ratio, shear modulus, and mode II fracture energy are determined in this bond-based peridynamic model by the Young's moduli and mode I fracture energies. These limitations are removed if one uses the state-based peridynamics (Silling et al., 2007). With the state-based peridynamic composite model one can model a composite

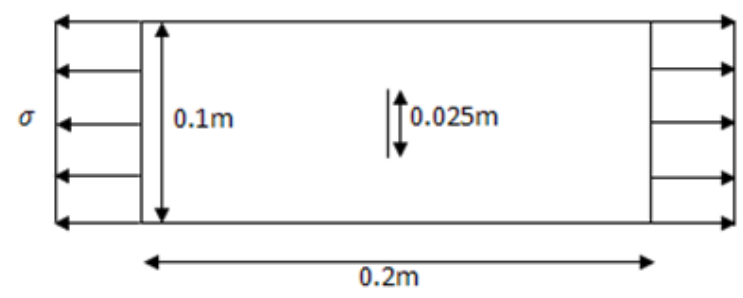

FIG. 5: Problem setup for the dynamic tensions test of a 0-degree UD composite with a center notch. 
TABLE 2: Material properties

\begin{tabular}{|c|c|}
\hline Property & Unidirectional \\
\hline Longitudinal Young's modulus $\mathrm{E}_{11}(\mathrm{GPa})$ & 329 \\
\hline Transverse Young's modulus $\mathrm{E}_{22}(\mathrm{GPa})$ & 6 \\
\hline Shear modulus $\mathrm{E}_{12}(\mathrm{GPa})$ & 4.4 \\
\hline Poisson's ratio $v_{12}$ & 0.346 \\
\hline Density $\rho\left(\mathrm{kg} / \mathrm{m}^{3}\right)$ & 1630 \\
\hline Fracture energy $\mathrm{G}_{0}^{11}\left(\mathrm{KJ} / \mathrm{m}^{2}\right)$ & 15.49 \\
\hline Fracture energy $\mathrm{G}_{0}^{22}\left(\mathrm{KJ} / \mathrm{m}^{2}\right)$ & 0.168 \\
\hline
\end{tabular}

material with arbitrary material properties. We use here the somewhat simpler bond-based version because our focus is on demonstrating the capabilities of the peridynamic approach in modeling dynamic fracture in a fiber-reinforced composite.

\subsection{The Elastic Strain Energy for the Scaled and Non-Scaled Models}

In this section we compare the total elastic strain energy of the scaled model and the non-scaled model with the exact classical elasticity solution. The elastic strain energy of a composite lamina with $0^{\circ}$ fiber orientation without a per-crack is examined for the homogeneous deformation (constant bi-axial strain $\alpha=0.005$ ) given below:

$$
\mathbf{F}=\alpha \mathbf{I}
$$

where $\mathbf{F}$, and $\mathbf{I}$ are deformation gradient tensor and identity tensor, respectively, and $\alpha$ is a constant positive value. The total strain energy for the rectangular plate (without the center cut) shown in Fig. 5 is the integral over the plate area of the nodal strain energy density. Since we are employing an approximate scheme for the spatial integration (see the algorithm for partial area evaluation in Bobaru and Ha, 2011; Ha et al., 2010b), we will only obtain approximations of the exact classical strain energy density. Moreover, because of the "skin effect" near the boundaries (see, e.g., Ha and Bobaru, 2011) additional error will be introduced. Nevertheless, as $m$ increases the approximate numerical integration approaches the exact integration, while decreasing the horizon $\delta$ the skin effect is reduced as well. The results for both the scaled [see Eq. (26)] and non-scaled [directly use Eqs. (17) and (18)] model are given in Table 3. The exact classical strain energy with the same homogeneous deformation and configuration, which can be obtained from Eq. (13), is $84.92 \mathrm{KJ}$.

For all results obtained from here on we employ the scaled model. For a computationally efficient solution one needs to use small values of $m$. A value of $m=5$ seems a reasonable compromise between efficiency and accuracy (relative to the classical model).

TABLE 3: Comparison of strain energy between scaled and non-scaled model with conical micromodulus function

\begin{tabular}{|c|c|c|c|c|c|c|}
\hline \multirow{2}{*}{ Model } & \multicolumn{2}{|c|}{$\delta=4 \mathbf{m m}$} & \multicolumn{2}{c|}{$\delta=2 \mathbf{m m}$} & \multicolumn{2}{c|}{$\delta=1 \mathbf{m m}$} \\
\cline { 2 - 7 } & SE-S $^{\mathbf{(}}(\mathbf{K J})$ & SE-NS $^{2}(\mathbf{K J})$ & SE-S $($ KJ) & SE-NS (KJ) & SE-S $($ KJ) & SE-NS (KJ) \\
\hline$m=3$ & 72.93 & 16.86 & 73.37 & 16.97 & 73.63 & 17.05 \\
\hline$m=6$ & 80.79 & 10.16 & 81.33 & 10.24 & 81.60 & 10.28 \\
\hline$m=12$ & 83.07 & 6.16 & 83.57 & 6.21 & 83.82 & 6.23 \\
\hline
\end{tabular}

${ }^{1}$ SE-S: Strain energy with the scaled model.

${ }^{2}$ SE-NS: Strain energy with the non-scaled model. 


\section{$5.3 m$-Convergence for the Crack Path}

We perform $m$-convergence for two different horizon sizes: $\delta=4 \mathrm{~mm}$ and $\delta=2 \mathrm{~mm}$. All of the models use uniform grid spacing. The peridynamic models for horizon $\delta=4 \mathrm{~mm}$ use $m=3$ or $\Delta x=1.33 \mathrm{~mm}$ ( 11,552 nodes $), m=6$ or $\Delta x=0.67 \mathrm{~mm}$ (45,602 nodes), and $m=12$ or $\Delta x=0.33 \mathrm{~mm}$ (181,202 nodes). The results in terms of the damage maps are shown in Fig. 6. We observe that the crack paths (seen as zones with non-zero damage) are similar for the different grids used and that the splitting fracture mode is captured for this lamina with $0^{\circ}$ fibers. We also mention that the damage takes place only in the matrix bonds; no fiber bonds break under the applied shock loading $\sigma=40$ Pa.

The damage index for a node is defined as the number of currently broken bonds by the initial number of bonds associated with that node. The expression for the damage index is, therefore,

$$
\mathrm{d}=\frac{n_{\text {broken }}}{n}
$$

where $n_{\text {broken }}$ and $n$ are the number of broken bonds and the number of initial bonds at a node. The damage index is a number between 0 and 1 ("0" means no bonds are broken, and " 1 " means all of the bonds are broken). Note that a damage index of around 0.4-0.5 may indicate that a fracture surface exists.

For horizon $\delta=2 \mathrm{~mm}$, the grids used are for $m=3$ or $\Delta x=0.67 \mathrm{~mm}$ (45,602 nodes), $m=4$ or $\Delta x=0.5 \mathrm{~mm}$ (80,802 nodes), and $m=8$ or $\Delta x=0.25 \mathrm{~mm}$ (321,602 nodes). The results are given in Fig. 7. The difference between the case with $m=4$ and $m=8$ is minimal. The splitting fracture mode is again captured well in this UD composite lamina with $0^{\circ}$ fibers. Also, no fiber failure occurs in these simulations. In Fig. 7(a), we use $m=3$ instead of $m=2$ because the number of nodes inside the horizon for $m=2$ is not sufficient to allow a sufficiently large number of directions in which a crack can grow as a result of bond breaking.

The above results indicate that using $m=5$ is a good choice since the crack path does not suffer significant changes if one uses an even denser grid, and this choice is computationally efficient, too. For all the remaining studies below we use $m=5$.

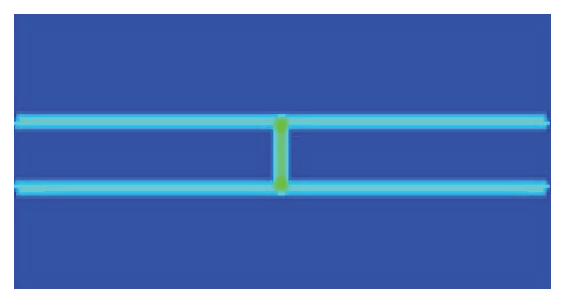

(a)

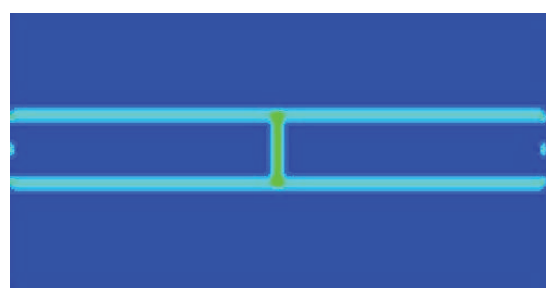

(b)

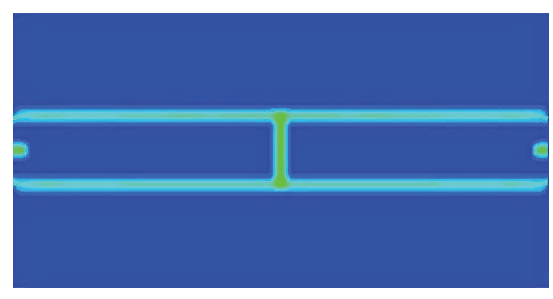

(c)

FIG. 6: Damage index maps (or crack path) computed with different grids for $\delta=4 \mathrm{~mm}$ at $50 \mu \mathrm{s}$ : (a) $\mathrm{m}=3$; (b) $m=4$; (c) $m=8$. The same damage index scale is used in all plots. 


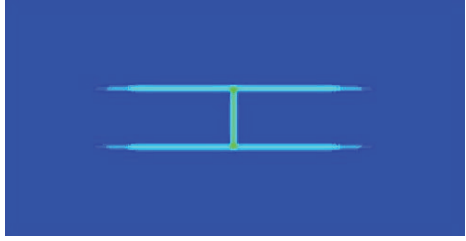

(a)

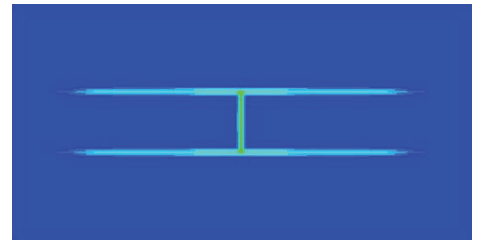

(b)

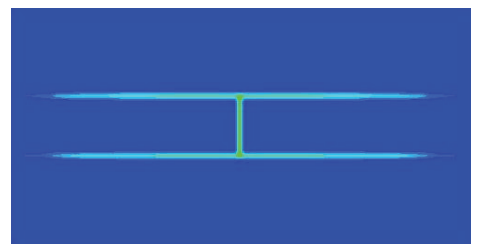

(c)

FIG. 7: Damage index maps (indicating the crack paths) computed with different grids for $\delta=2 \mathrm{~mm}$ at $50 \mu \mathrm{s}$ : (a) $m=3$; (b) $m=4$; (c) $m=8$

\section{4 -Convergence Results}

As mentioned by Ha and Bobaru (2010), the size of initial damage or notch area changes when the horizon size $\delta$ changes, and the damaged area becomes smaller as the horizon decreases. The $\delta$-convergence study below has to be understood in this context; note that the critical relative elongation also changes with respect to the changing horizon [as seen from Eq. (22)].

For a fixed value $m=5$, we use four different kinds of horizon sizes (uniform grid spacing): $\delta=4 \mathrm{~mm}$ or $\Delta x=0.8 \mathrm{~mm}$ (31,752 nodes), $\delta=3 \mathrm{~mm}$ or $\Delta x=0.6 \mathrm{~mm}(56,112$ nodes $), \delta=2 \mathrm{~mm}$ or $\Delta x=0.4 \mathrm{~mm}(126,002$ nodes), and $\delta=1 \mathrm{~mm}$ or $\Delta x=0.2 \mathrm{~mm}(50,2002)$. We note that for $m=5$ and $\delta=1 \mathrm{~mm}$ the total elastic strain energy computed with peridynamics is only about $4 \%$ different from the classical exact value.

The results showing the damage map for each case are given in Fig. 8. In all simulations the symmetric path of splitting fracture mode is obtained and only matrix bond breakage is observed, no fiber bonds break (besides those corresponding to the initial center cut). The damage patterns from these peridynamic simulations agree very well with the experimental observations given by Bogert et al. (2006), who also report only matrix breaking taking place (under quasi-static loadings). Notice in Fig. 8 that the crack length changes during the $\delta$-convergence study because the crack speed changes as the horizon becomes smaller. It is noted that the largest horizon used $(\delta=4)$ results in a strong nonlocal effect since the size of the center cut can be, in this case, covered by only about three horizon regions. The "large" nonlocality induces a much higher crack propagation speed than realistically expected. The crack propagation speed from our $\delta$-convergence results and comparisons with analytical solutions for the classical steady-state dynamic debonding crack propagation are discussed in the next section.

\subsection{Study of the Splitting Crack Propagation Speed}

In this section we study the crack propagation speed in terms of the horizon size for the splitting fracture mode. The details of computing the crack propagation speed in peridynamics can be found in the study done by Ha and Bobaru (2010).

We could use a larger and wider panel to see if the crack propagation speed approaches a steady-state velocity before it starts interacting with the waves reflected from the boundary. However, that would require a significant 


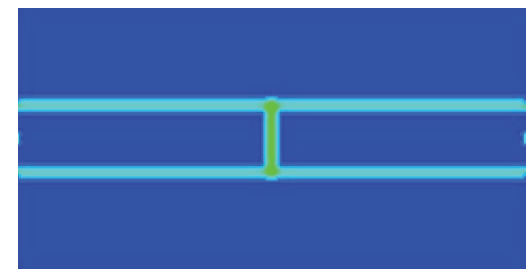

(a)

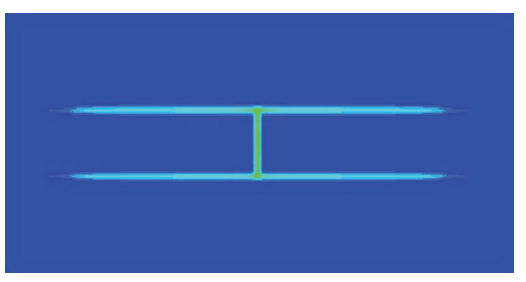

(c)

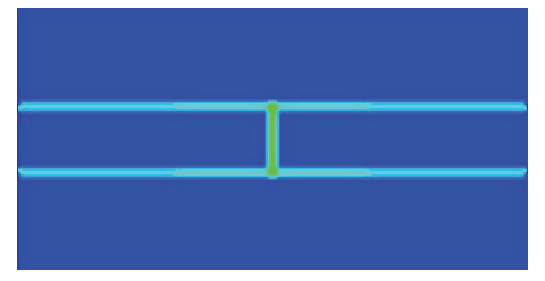

(b)

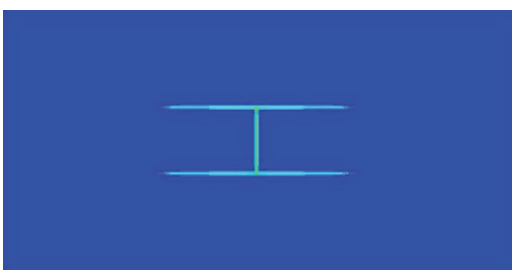

(d)

FIG. 8: Damage index maps computed with various $\delta(m=5)$ at $50 \mu \mathrm{s}$ : (a) $\delta=4 \mathrm{~mm}$; (b) $\delta=3 \mathrm{~mm}$; (c) $\delta=2 \mathrm{~mm}$; (d) $\delta=1 \mathrm{~mm}$

increase in the computational resources. In the future, we will run simulations with a larger and wider panel, but with a parallelized code.

As shown in Fig. 9, the crack starts propagating at about $22 \mu$ s after the loads have been applied at the boundaries (see Fig. 5). The larger horizon size simulation shows that the crack already reaches the boundaries at around $44 \mu$ s. As the horizon size decreases, the average and the maximum crack propagation speeds decrease as well. The stress waves strongly affect the crack propagation speed. The observed speed-up and slow-down of the crack propagation speed seen in Fig. 9 correlate well with the propagation of the stress/strain waves in the material, as seen from Fig. 10.

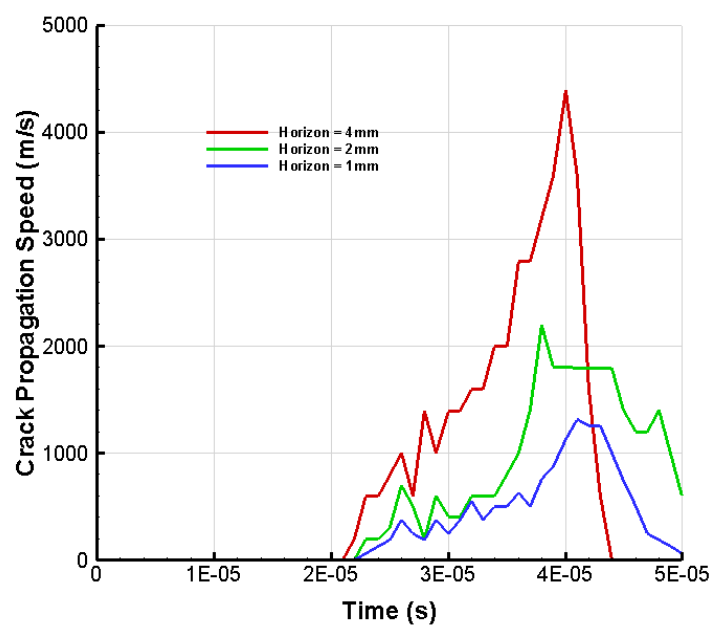

FIG. 9: Crack propagation speed for different horizons 


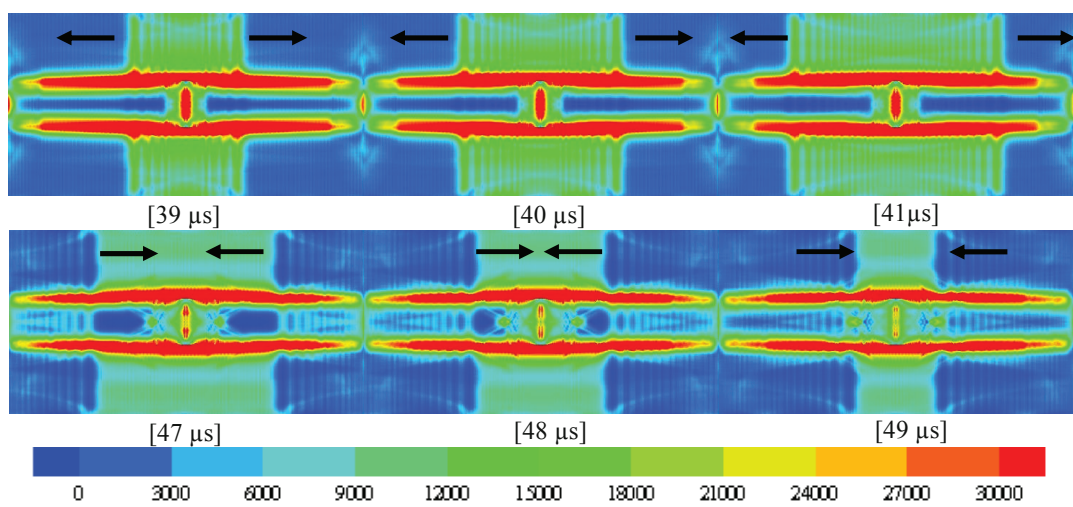

FIG. 10: Strain energy density $(\delta=1 \mathrm{~mm}$ and $m=5)$ at different times (black arrows indicate the direction of motion of the stress waves). The crack path has not yet reached the state shown in Fig. 8(d), which was obtained at $50 \mu \mathrm{s}$.

In Fig. 10 we show the strain energy density at different times for the case with the smallest horizon used in this paper. It is apparent that when the stress waves are moving away from the crack tip, the crack propagation speed increases, and when the reflected waves return and meet the crack tip, the crack propagation speed decreases. Because of the dynamic loading and interaction with the stress waves, the crack arrests temporarily before it continues to propagate again.

We focus next on analyzing the maximum splitting crack propagation speed. A theoretical framework has been established by Yang et al. (1991) for interface fracture mechanics, which includes dynamic crack propagation and strain-rate-dependent material response. Yang et al. (1991) argue that for cracks running at non-uniform speed, the near tip singularity is 2D, and is instantaneously in a steady state. A formula for the propagation speed of a debonding crack is given. Tsai et al. (2001) used this formulation and found that the dynamic fracture toughness is roughly equal to the static fracture toughness and is not significantly influenced by crack speeds up to $1100 \mathrm{~m} / \mathrm{s}$. In particular, modes I and II energy release rates remain nearly constant when the crack propagation speed is up to about $50 \%$ of the Rayleigh wave speed for S2/8553 glass/epoxy and AS4/3501-6 carbon/epoxy composites.

The crack propagation speed for a moving crack in a homogeneous orthotropic material can be obtained from the energy release rate and stress intensity factor (Yang et al., 1991; Tsai et al., 2001). The crack propagation speed for the splitting mode can be written as follows:

$$
\mathrm{G}_{I I}=\frac{1}{2} \mathrm{H}_{11} \mathrm{~K}_{I I}^{2}
$$

where $\mathrm{G}_{I I}$ and $\mathrm{K}_{I I}$ are the fracture energies for mode II fracture and the mode II stress intensity factor. The expression of $\mathrm{H}_{11}$ is given as

$$
\mathrm{H}_{11}=\frac{1}{\mathrm{C}_{66} \mathrm{R}} \rho_{22} \alpha_{2}^{2}[2(1+\mathrm{s}) / \xi]^{1 / 2}
$$

where

$$
\begin{aligned}
& \rho_{11}=\frac{\mathrm{C}_{11}}{\mathrm{C}_{66}}, \quad \rho_{22}=\frac{\mathrm{C}_{22}}{\mathrm{C}_{66}}, \quad \rho_{12}=\frac{\mathrm{C}_{12}}{\mathrm{C}_{66}}, \alpha_{1}=\sqrt{\left(1-\frac{\rho v_{\mathrm{e}}^{2}}{\mathrm{C}_{11}}\right)}, \quad \alpha_{2}=\sqrt{\left(1-\frac{\rho v_{\mathrm{e}}^{2}}{\mathrm{C}_{66}}\right)} \\
& \xi=\alpha_{1} \alpha_{2} \sqrt{\rho_{11} / \rho_{22}}, \quad \mathrm{~s}=\frac{\alpha_{2}^{2}+\rho_{11} \rho_{22} \alpha_{1}^{2}-\left(1+\rho_{12}\right)^{2}}{2 \alpha_{1} \alpha_{2} \sqrt{\rho_{11} \rho_{22}}}, \quad \mathrm{R}=\rho_{22}\left(\rho_{22} \xi-1+\alpha_{2}^{2}\right)-\rho_{12}^{2} \alpha_{2}^{2} / \xi
\end{aligned}
$$

where $\mathrm{C}_{11}, \mathrm{C}_{22}, \mathrm{C}_{66}$, and $\mathrm{C}_{12}$ are the elastic constants defined in Section 3 ; and $\rho$ and $\mathrm{v}_{\mathrm{e}}$ are the density and the crack propagation speed for certain values of fracture energy and the stress intensity factor, respectively. 
With all of the parameters provided as given by Jose et al. (2001), the crack propagation speed of the splitting mode for the values of $\mathrm{K}_{I I}$ and $\mathrm{G}_{I I}$ [from Eqs. (31) and (32)] is $\sim 820 \mathrm{~m} / \mathrm{s}$. For the M55J/M18 carbon/epoxy composite, the Rayleigh wave speed is about $1700 \mathrm{~m} / \mathrm{s}$. In general, the fracture energy depends on the crack propagation speed. However, as pointed out by Tsai et al. (2001), the variation is minimal for cracks moving at speeds lower than $50 \%$ Rayleigh wave speed. Therefore, it is reasonable to consider the fracture energy constant. In our peridynamic computations we use constant fracture energy in the formulas that give the critical relative elongation of the bonds. The values used in the peridynamic computations for mode I fracture energy in the $0^{\circ}$ and $90^{\circ}$ orientations are given in Table 2.

The relative error for the crack propagation speed computed with peridynamics for the different horizon sizes, against the analytical value mentioned above, is

$$
\mathrm{R}_{\text {error }}=\frac{\mathrm{v}-\mathrm{v}_{\mathrm{e}}}{\mathrm{v}_{\mathrm{e}}}
$$

where $\mathrm{v}$ and $\mathrm{v}_{\mathrm{e}}$ are the maximum crack propagation speed from the peridynamic computations and the analytical crack propagation speed computed above, respectively. In Fig. 11(a), we give the relative error versus the horizon size and in Fig. 11(b) the same plot is given in a log-log scale.

In the log-log plot the results indicate a linear relationship. With a linear curve fit, we can obtain the crack speed for any horizon size as follows:

$$
\mathrm{v}_{\delta}=\mathrm{v}_{\mathrm{e}} \mathrm{e}^{\alpha}+\mathrm{v}_{\mathrm{e}}
$$

where

$$
\alpha=\mathrm{k} * \log \left(\frac{1}{\delta}\right)-0.49
$$

where $\mathrm{v}_{\delta}$ is the crack propagation speed for a given horizon size $\delta$, and $\mathrm{k}$ is the slope of the curve-fit line and equals -1.45 when the propagation speeds for the horizons equal to $4 \mathrm{~mm}, 2 \mathrm{~mm}$, and $1 \mathrm{~mm}$ were used. We compute the crack propagation speed for a value of the horizon equal to $3 \mathrm{~mm}$. The maximum crack propagation speed from peridynamics calculations with $\delta=3 \mathrm{~mm}$ is around $3,420 \mathrm{~m} / \mathrm{s}$ while the value obtained from the linear curve fit above for the same horizon size is $3,281 \mathrm{~m} / \mathrm{s}$. The relative difference between these two results is less than $5 \%$.

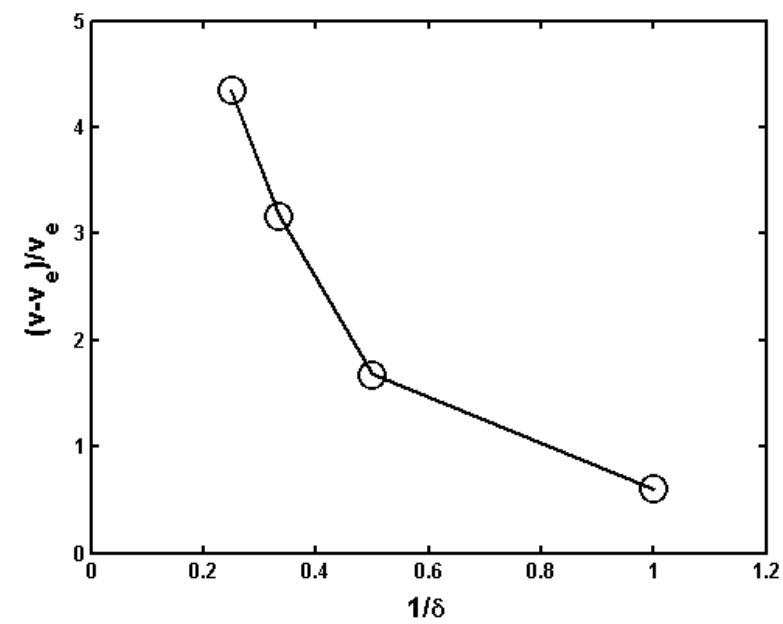

(a)

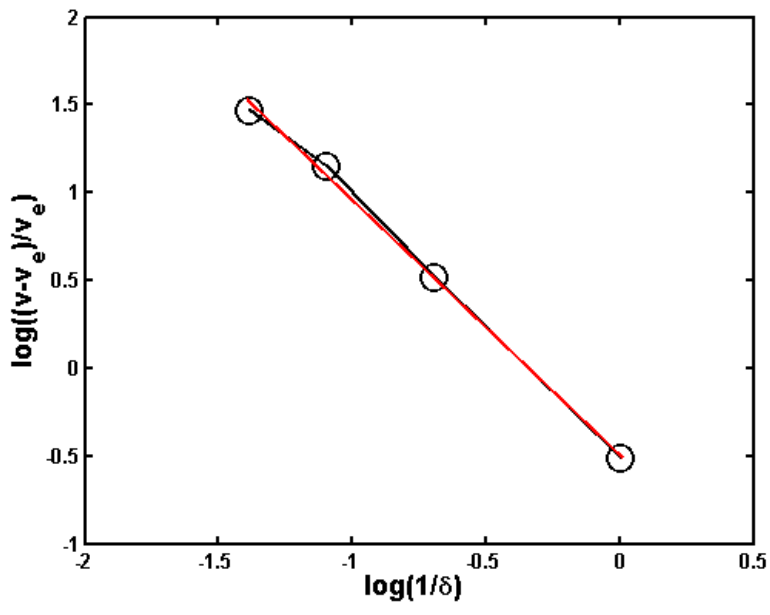

(b)

FIG. 11: Relative difference of the maximum crack propagation speed from the peridynamic computations for different horizon sizes versus the analytical value (a); linear curve fit in the log-log scale for the data on the left (b) 
It appears that the maximum crack propagation speed computed by peridynamics, in the limit of the horizon going to zero, converges to the value computed analytically from the classical model for the "same" composite material. For example, if we choose a horizon size of about $0.15 \mathrm{~mm}$, the crack propagation speed obtained from Eq. (34) is about $851 \mathrm{~m} / \mathrm{s}$. The relative difference between this value and the analytical results $(820 \mathrm{~m} / \mathrm{s})$ is about $3.6 \%$. Note an important difference between the parameters used in the analytical formula and those used in our peridynamic computations: in the analytical classical approach, the value for $\mathrm{G}_{I I}$ is prescribed, while in our model we input $\mathrm{G}_{I}$ for $0^{\circ}$ and $90^{\circ}$ fiber orientations. The $\mathrm{G}_{I I}$ value used in Eq. (31) from Jose et al. (2001) falls between the two $\mathrm{G}_{I}$ values used in the peridynamic computations (see Table 2).

The results show that a sub-millimeter horizon produces maximum crack propagation speed values in this splitting fracture mode for the given lamina geometry under the given dynamic loading conditions, close to those given by the classical analytical model.

\subsection{Multiple Splitting Cracks under Higher Loading}

We use the same material and problem configuration but now a higher uniform tensile load $\sigma=400 \mathrm{~Pa}$ is applied abruptly and kept constant in time after that. In the plots given in Figs. 12(a)-(c) we observe the evolution in time of damage patterns in the matrix and in Fig. 12(d) we show the damage in the fiber bonds only. We note that no fibers are broken besides those corresponding to the original center cut. The damage in the matrix bonds spreads toward the horizontal center line of the lamina. Note that initially, a single splitting takes place, but as the waves reflect back and forth from the boundaries, multiple splitting failures ensue.

To monitor the fracture behavior of the fiber bonds we define the fiber damage index of a node as the number of broken fiber bonds by the original number of such bonds for that node:

$$
\mathrm{d}_{\mathrm{fiber}}=\frac{n_{\mathrm{fb}}^{\text {broken }}}{n_{\mathrm{fb}}}
$$

where $n_{\mathrm{fb}}^{\text {broken }}$ and $n_{\mathrm{fb}}$ are the number of broken peridynamic fiber bonds and the original number of fiber bonds, respectively. This "fiber damage index" also ranges between 0 and 1 (" 0 " means no peridynamic fiber bonds are broken; "1" means all fiber bonds are broken).

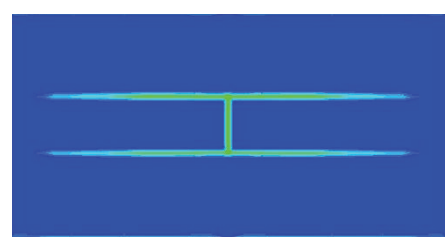

(a)

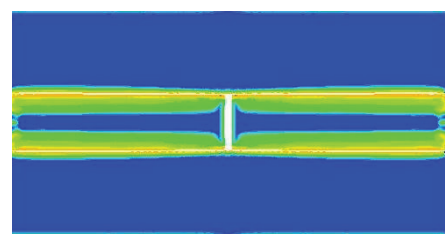

(c)

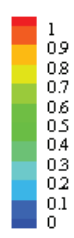

(d)

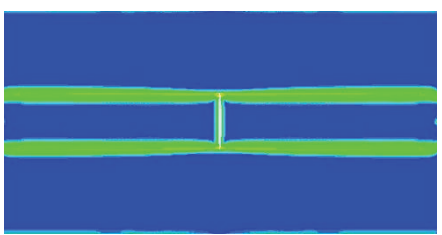

(b)

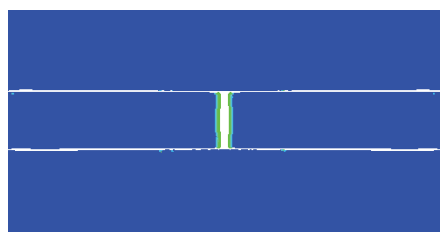

FIG. 12: Damage maps under higher loading conditions: (a) single splitting forms (at $15 \mu \mathrm{s}$ ); (b) multiple splits develop (at $30 \mu \mathrm{s}$ ); (c) final failure $(50 \mu \mathrm{s})$; (d) damage map for the fiber bonds alone at $50 \mu \mathrm{s}$ (no fiber breakage other than the initial notch; the green area represents the initial notch). Notice that material separation happens since the matrix bonds are broken. 


\section{CONCLUSIONS}

In this paper, we proposed a new homogenized multiscale peridynamic model for simulating fracture in a UD fiberreinforced composite lamina. Analytical expressions for the micromodulus function and the critical relative elongation are obtained by calibrating them, for a given horizon size, to measurable material properties of the UD composite lamina, such as the elastic strain energy density in a homogeneous deformation and mode I fracture energies. We also provide formulas for scaling factors corresponding to a discretized model.

The splitting fracture mode for a UD composite lamina with $0^{\circ}$ fiber orientation is easily captured by our peridynamic model. In the bond-based peridynamic model only Young's modulus for the longitudinal and transverse directions, and modes I fracture energies for the longitudinal and transverse directions, are used. The mode II fracture energy and the Poisson ratio are implicit in this model. The results agree very well with experimental observations published in the literature. The crack propagation path for both $\delta$-convergence and $m$-convergence is symmetric, and parallel to the loading direction, reflecting the splitting fracture mode. For the particular composite material used here, no fiber breakage is observed. We notice that because of the dynamic loading the crack propagation speed is strongly affected by the stress waves in the body.

We also compared the maximum crack propagation speed from the peridynamic simulations with an analytical solution for a steady-state dynamic debonding crack. The peridynamic solutions show a maximum crack propagation speed for the splitting mode that approaches the theoretical dynamic debonding solution when the horizon goes to zero. A sub-millimeter horizon is a reasonable one to use for this type of dynamic loading.

The model introduced in this paper and the results shown demonstrate that, contrary to some published remarks in the literature, it is possible to successfully use homogenization to model fracture and damage in fiber-reinforced composites using peridynamics. The peridynamic model for UD composite lamina introduced in this paper will be used in the future to study dynamic fracture of various fiber orientations and it will be extended to model dynamic fracture and damage in composite laminates.

\section{ACKNOWLEDGMENTS}

The authors are thankful for the financial support offered through research contracts between the University of Nebraska-Lincoln and Sandia National Laboratories (Dr. S. A. Silling), contract number 568428, Boeing Research \& Technology, the Applied Mathematics division (Dr. E. Askari), and the Army Research Office (Dr. B. LaMattina) and ARL (Dr. C. F. Yen), ARO award number 58450EG. We also acknowledge the discussions with Dr. Silling that have led to an improved paper. The detailed comments and suggestions from the two anonymous reviewers helped greatly to improve the paper.

\section{REFERENCES}

Bobaru, F., Influence of van der Waals forces on increasing the strength and toughness in dynamic fracture of nanofibre networks: A peridynamic approach, Modell. Simul. Mater. Sci. Eng., vol. 15, pp. 397-417, 2007.

Bobaru, F. and Ha, Y. D., Adaptive refinement and multiscale modeling in 2D peridynamics, Int. J. Multiscale Comp. Eng., advance online publication, DOI: 10.1615/IntJMultCompEng.2011002793, 2011.

Bobaru, F., Yang, M., Alves, L. F., Silling, S. A., Askari, E., and Xu, J., Convergence, adaptive refinement, and scaling in 1D peridynamics, Int. J. Numer. Methods Eng., vol. 77, no. 6, pp. 852-877, 2009.

Bogert, P. B., Satyanarayana, A., and Chunchu, P. B., Comparison of damage path predictions for composite laminates by explicit and standard finite element analysis tool, Proc. of 47th AIAA Structures, Structural Dynamics, and Materials Conference, Newport, RI, 2006.

Cristescu, N. D., Craciun, E. M., and Soos, E., Mechanics of Elastic Composite, New York: Chapman \& Hall/CRC, 2004.

Eskandari, E. and Nemes, J. A., Dynamic testing of composite laminates with a tensile split hopkinson bar, J. Compos. Mater., vol. 34, no. 4, pp. 260-273, 2000.

Ha, Y. D. and Bobaru, F., Traction boundary conditions in peridynamics: A convergence study, Technical Report, Department of Engineering Mechanics, University of Nebraska-Lincoln, Lincoln, NE, 2009. 
Ha, Y. D., and Bobaru, F., Studies of dynamic crack propagation and crack branching with peridynamics, Int. J. Fract., vol. 162, no. 1-2, pp. 229-244, 2010.

Ha, Y. D. and Bobaru, F., Characteristics of dynamic brittle fracture captured with peridynamics, Eng. Fract. Mech., vol. 78, no. 6, pp. 1156-1168, 2011a.

Ha, Y .D. and Bobaru, F., Dynamic brittle fracture captured with peridynamics, paper IMECE2011-65515, in Proc. of the ASME 2011 International Mechanical Engineering Congress \& Exposition, IMECE2011, November 11-17, 2011, Denver, Colorado, USA, $2011 b$.

Ha, Y. D., Hu, W., and Bobaru, F., The skin effect and numerical integration in peridynamics, Technical Report, Department of Engineering Mechanics, University of Nebraska-Lincoln, Lincoln, NE, 2010.

Hallett, S. R., Green, B. G., Jiang, W. G., and Wisnom, M. R., An experimental and numerical investigation into the damage mechanisms in notched composites, Compos. Part A: Appl. Sci. Manuf., vol. 40, no. 5, pp. 613-624, 2009.

Halpin, J. C. and Kardos, J. L., The Halpin-Tsai equations: A review, Polym. Eng. Sci., vol. 16, no. 5, pp. 344-352, 1976.

Hu, W., Ha, Y. D., and Bobaru, F., Peridynamic model for dynamic fracture in unidirectional fiber-reinforced composites, (submitted), 2011.

Jiang, W. G., Hallett, S. R., Green, B. G., and Wisnom, M. R., A concise interface constitutive law for analysis of delamination and splitting in composite materials and its application to scaled notched tensile specimens, Int. J. Numer. Methods Eng., vol. 69, no. 9, pp. 1982-1995, 2007.

Jose, S., Kumar, R. R., Jana, M. K., and Rao, G. V., Intralaminar fracture toughness of a cross-ply laminate and its constituent sub-laminates, Compos. Sci. Technol., vol. 61, no. 8, pp. 1115-1122, 2001.

Kazemahvazi, S., Zenkert, D., and Burman, M., Notch and strain rate sensitivity of non-crimp fabric composites, Compos. Sci. Technol., vol. 69, no. 6, pp. 793-800, 2009.

Kortschot, M. T. and Beaumont, P. W. R., Damage mechanics of composite materials. I: Measurements of damage and strength, Compos. Sci. Technol., vol. 39, no. 4, pp. 289-301, 1990a.

Kortschot, M. T. and Beaumont, P. W. R., Damage mechanics of composite materials. II: A damaged-based notched strength model, Compos. Sci. Technol., vol. 39, no. 4, pp. 303-326, 1990b.

Kilic, B., Agwai, A., and Madenci, E., Peridynamic theory for progressive damage prediction in center-cracked composite laminates, Compos. Struct., vol. 90, pp. 141-151, 2009.

Lee, D., Tippur, H., Kirugulige, M., and Bogert, P., Experimental study of dynamic crack growth in unidirectional graphite/epoxy composites using digital image correlation method and high-speed photography, J. Compos. Mater., vol. 47, no. 19, pp. 2081$2108,2009$.

Pineda, E. J., Waas, A. M., Bednarcyk, B. A., Collier, C. S., and Yarrington, P. W., Progressive damage and failure modeling in notched laminated fiber reinforced composites, Int. J. Fract., vol. 158, no. 2, pp. 125-143, 2009.

Ravi, S., Iyengar, N. G. R., Kishore, N. N., and Shukla, A., Experimental studies on damage growth in composite under dynamic loads, Appl. Compos. Mater., vol. 8, no. 2, pp. 79-97, 2001.

Silling, S. A., Reformulation of elasticity theory for discontinuities and long-range forces, J. Mech. Phys. Solids., vol. 48, no. 1, pp. 175-209, 2000.

Silling, S. A. and Askari, E., A meshfree method based on the peridynamic model of solid mechanics, Comput. Struct., vol. 83, no. 17-18, pp. 1526-1535, 2005.

Silling, S. A. and Bobaru, F., Peridynamic modeling of membranes and fibers, Int. J. Non-Linear Mech., vol. 40, pp. 395-409, 2005.

Silling, S. A., Zimmermann, M., and Abeyaratne, R., Deformation of a peridynamic bar, J Elast., vol. 73, pp.173-190, 2003.

Silling, S. A., Epton, M., Weckner, O., Xu, J., and Askari, E., Peridynamic states and constitutive modeling, J. Elast., vol. 88, no. 2, pp. 151-184, 2007.

Tsai, J. L., Guo, C., and Sun, C. T., Dynamic delamination fracture toughness in unidirectional polymeric composites, Compos. Sci. Technol., vol. 61, no. 1, pp. 87-94, 2001.

Wu, E. M., Fracture mechanics of anisotropic plates, Composite Materials Workshop, pp. 20-43, Lancaster, PA: Technomic Publishing, 1968.

Xu, J., Askari, E., Weckner, O., Razi, H., and Silling, S. A., Damage and failure analysis of composite laminates under biaxial loads, Proc. of 47th AIAA Structures, Structural Dynamics, and Materials Conference, Honolulu, 2007.

Xu, J., Askari, E., Weckner, O., and Silling, S. A., Peridynamic analysis of impact damage in composite laminates, J. Aerosp. Eng., vol. 21, no. 3, pp. 187-194, 2008.

Yang, W., Suo, Z., and Shih, C. F., Mechanics of dynamic debonding, Proc. R. Soc. London, Ser. A, vol. 433, pp. 679-697, 1991. 